\title{
RECENT ADVANCES IN RADAR TECHNOLOGY AND TECHNIQUES FOR AFFORDABLE PLANETARY REMOTE SENSING
}

\author{
Jakob van Zyl, Charles Elachi, and Yunjin Kim \\ Jet Propulsion Laboratory \\ California Institute of Technology \\ Pasadena, CA, USA
}

\section{INTRODUCTION}

Radar remote sensing instruments acquire data useful for geophysical investigations by measuring electromagnetic interactions with natural objects[1]. Examples of radar remote sensing instruments include synthetic aperture radars (SAR), scatterometers, altimeters, and radar sounders. The main advantage of radar instruments is their ability to penetrate clouds, rain, tree canopies, and even dry soil surfaces depending upon the operating frequencies. In addition, since a remote sensing radar is an active instrument, it can operate during day and night by providing its own illumination.

Imaging remote sensing radars such as SAR produce high resolution (from sub meter to few tens of meters) images of surfaces. The geophysical information can be derived from these high resolution images by using proper post processing techniques. Scatterometers measure the backscattering cross section accurately in order to characterize surface properties such as roughness. Altimeters are used to obtain accurate surface height maps by measuring the round trip time delay from a radar sensor to the surface. Radar sounders can image underground material variations by penetrating deeply into the ground. Unlike surveillance radars, remote sensing radars require accurate calibration in order for the data to be useful for scientific applications.

The past decade saw a dramatic increase in the capabilities of radar remote sensing instruments. Advanced techniques such as polarimetry[2] and interferometry[3] were introduced for SAR systems, and scatterometers and altimeters moved from demonstration to operational instruments.

In this paper we start with a short description of a few mission concepts that exemplify the types of techniques and technologies that will be required for future missions. These are only examples, and do not represent a complete set of future mission concepts. Some missions are operational, and some are only in the concept phase, and are included to illustrate the techniques and technologies required to enable these measurements. The mission descriptions are followed by a description of the current progress in radar technology development.

\section{RADAR MISSIONS AND INSTRUMENTS}

\section{I Planetary radar instruments}

The MARSIS (Mars Advanced Radar for Subsurface and Ionospheric Sounding) instrument, scheduled to be flown on the ESA Mars Express spacecraft to orbit the planet Mars in 2004, is a low-frequency nadirlooking pulse limited radar sounder and altimeter with ground penetration capabilities, which uses synthetic aperture techniques and a secondary receiving antenna to isolate subsurface reflections. The main purpose of the MARSIS instrument is to search for the presence of liquid water under the Martian surface. The MARSIS instrument consists of a single transmitter, and two antennas, a nadirlooking dipole and a nadir looking monopole, each connected to a receiver. The transmitted signal is radiated through the nadir looking dipole antenna. After the transmit pulse is completed, the return signal from the Martian surface is received by both antennas. The signals are then routed to the two receivers where they are amplified and downconverted to range offset video prior to conversion to digital form. The secondary monopole antenna, oriented along the nadir axis, will receive mostly the off-nadir surface returns, that could be thus subtracted in the ground re-processing, further reducing the surface clutter level (about 15-20 dB). The MARSIS instrument will operate in the frequency range of approximately 1-5 $\mathrm{MHz}$.

Low frequency radar sounding is also one of the leading techniques planned to be used in the search for a liquid ocean on Europa, one of the moons of Jupiter. Ever since the initial observation of Europa by the Voyager spacecraft, it has been suggested that there may be a liquid water ocean beneath Europa's icy shell. Since liquid water is essential for life, the existence of a liquid water ocean is of great interest. Recent high resolution images of the Europa surface from the Galileo spacecraft also suggest the existence of a liquid ocean. In order to detect the liquid ocean under the ice of Europa, a radar sounder mission was suggested, and the concept was studied by the Europa sounder instrument definition team.

The primary objective of the Europa radar sounder is to characterize the ice volume beneath Europa's ice shell. It is well known that radar at frequencies of $10-100 \mathrm{MHz}$ can be used to map ice thickness on Earth. If the Europa 
ice shell exhibit properties similar to the ice on Earth, a radar sounder may be the most effective tool for this study that requires sounding down the tens of kilometers. The Europa sounder concept uses a lighweight Yagi antenna to achieve a narrow beam. This narrow beam pattern significantly reduces the off-nadir returns from the surface which would otherwise be misinterpreted as sub-surface echoes.

\subsection{Earth science missions}

Earth science missions can be broadly separated into two categories: high resolution imagers with limited spatial coverage, and low resolution imagers with large spatial coverage. The former is mainly used for local to regional studies of slowly changing phenomena, while the latter is used for regional to global studies of phenomena that exhibit significant temporal dynamics.

Examples of current low resolution radar missions include the TOPEX/Poseidon radar altimeter mission to measure ocean surface height, now famous for the data provide on the $\mathrm{El}$ Nino and La Nina cycles. The TOPEX/Poseidon altimeter, launched in 1992, has been shown to measure the ocean surface topography accurately to about $4 \mathrm{~cm}$ at a resolution of about $13 \mathrm{~km}$ with a ten day repeat cycle. These measurements will be continued by the Jason-1 mission, now scheduled for launch in late 2000 .

The main drawback of the TOPEX - style altimeter missions is the fact that it measures only a profile of ocean topography directly underneath the spacecraft. This leaves gaps of $\sim 300 \mathrm{~km}$ between tracks on the equator. The next generation of ocean altimeters, proposed for the Jason-2 mission, will largely overcome this drawback by using a low resolution radar interferometer for measuring the ocean surface topography. This instrument will use two antennas, separated by approximately $10 \mathrm{~m}$, to form a radar interferometer that would simultaneously image a swath of approximately $75 \mathrm{~km}$ on both sides of the spacecraft nadir track. While this still does not provide full global coverage, it increases the amount of coverage by several orders of magnitude over previous missions. In addition, since a two-dimensional picture is acquired rather than a single profile, much smaller phenomena, such as large scale eddies, can now be studied, giving a more complete picture of ocean circulation.

Another example of a low resolution radar mission is the QuikScat scatterometer launched in June 1999 and currently used to measure ocean winds. QuikScat was place in orbit to continue measurements started by the NSCAT instrument. Unfortunately the latter ceased operating after less than one year following the premature demise of the ADEOS spacecraft. The NSCAT instrument was a classical radar scatterometer implemented with socalled stick antennas illuminating each side of the spacecraft nadir track, realizing a swath of approximately $600 \mathrm{~km}$ on each side of the spacecraft with a gap between the swaths of approximately $350 \mathrm{~km}$. The QuikScat implementation uses an alternative implementation of a spinning antenna to acquire data in a conical scanning pattern. This results in a large swath of approximately $1800 \mathrm{~km}$ with no gaps along the nadir line. An additional attractive feature is that all data are acquired at a fixed incidence angle, making data reduction significantly easier. The Quikscat implementation uses an antenna of approximately $1 \mathrm{~m}$ in diameter, resulting in resolutions of approximately $25-50 \mathrm{~km}$ after data processing. Future systems are being studied which would reduce the resolution by roughly an order of magnitude.

A third class of low resolution radar systems are proposed for studies of the freeze/thaw and hydrologic cycle. For these types of studies, near global coverage is required every few $(-3-5)$ days at resolutions on the order of $1 \mathrm{~km}$. Furthermore, to sense to a reasonable depth, these missions require lower frequency systems - on the order of $1 \mathrm{GHz}$ or lower. For these applications, scatterometer antennas will become unrealistically large, and low resolution synthetic aperture radar (SAR) systems are being considered. The wide swath required, however, (on the order of $600-900 \mathrm{~km}$ ) makes the implementation of these systems very challenging. If the traditional ScanSAR implementation is used, the antenna required is on the order of $10 \mathrm{~m}$ by $3 \mathrm{~m}$ in size, and very lightweight transmit/receive modules and antenna structures are required. A different implementation approach uses a narrow (on the order of $0.5-0.75 \mathrm{~m}$ ) antenna that is very long (on the order of $30 \mathrm{~m}$ or more). With this implementation, no elevation phase steering is required, but the long antenna is required to reduce the range ambiguities to an acceptable level. The best candidate for this type of antenna is an inflatable structure.

Many other Earth science studies require images with resolutions on the order of $10-50 \mathrm{~m}$ of relatively small areas, on the order of a few hundred kilometers on a side. One example of such an application is the study of surface deformation due to seismic or volcanic activity.

These studies require images acquired from nearly the same vantage point ever few (5-10) days. These images are then processed interferometrically to extract information about the surface deformation. This type of mission requires antennas of "traditional" size, about $10 \mathrm{~m} \mathrm{x} 3 \mathrm{~m}$ for L-band. Therefore, one of the main drivers of the mission cost is again the mass of the transmit/receive modules and the antenna and its associated structure. Lightweight antenna technology is most important. Another very important requirement is the fact that the images must be acquired 
from nearly the same vantage point to reduce the effect of local relief on the measurement of the surface deformation. Accurate navigation is therefore essential, and usually it is desired to fly the spacecraft in "tube" of approximately 250 $\mathrm{m}$ diameter for an $\mathrm{L}$-band radar.

Radar interferometry has been shown to be a very cost effective tool for measuring digital topography. The Shuttle Radar Topography Mission (SRTM) is capable of producing a three-dimensional image of $80 \%$ of the Earth's land surface in a single 10-day Space Shuttle flight [4]. It is the first spaceborne single-pass interferometric SAR and will produce the first near-global, high-resolution, digital elevation map. Such a global map will be constructed significantly sooner than with other systems by taking advantage of the unique opportunity offered through augmentation of the previously flown NASA SIR-C and the $\mathrm{X}-\mathrm{SAR}$. Addition of a C-band receive antenna, extended from the Shuttle bay on a mast and operating in concert with the existing SIR-C antenna, forms an interferometric pair. Due to the practical limitation of approximately 60 meters for the mast length, the shorter $\mathrm{C}$-band wavelength produced greater height accuracy than with the SIR-C Lband. C-band allowed a smaller antenna, which was more manageable for the mast. The data channels, previously used for L-band, could be used to support the added interferometric receive channels through frequency conversion. The existing SIR-C SCANSAR mode, combined with the dual-polarization capability, allowed a $225-\mathrm{km}$ swath at $\mathrm{C}$-band. This provided complete land coverage within 57 degrees north and south latitude. It is estimated that the achievable height accuracy is less than 16 meters absolute and 11 meters relative, where these values are the $90 \%$ linear error after processing both ascending and descending passes. The elevation postings are 30 meters.

Other Earth science studies require polarimetric radar data. Electromagnetic wave propagation is a vector phenomenon, so that measurement of the complete complex scattering matrix is necessary to completely characterize the scattering properties of terrain. The typical implementation of a radar polarimeter involves transmitting a wave of one polarization and receiving echoes in two orthogonal polarizations simultaneously. This is followed by transmitting a wave with a second polarization, and again receiving echoes with both polarizations simultaneously. In this way, all four elements of the scattering matrix is measured. The NASA/JPL AIRSAR system pioneered this implementation for $\mathrm{SAR}$ systems, and the same implementation was used in the SIR-C part of the SIR-C/XSAR radars. Polarimetric SAR data are particularly useful in the study of vegetation dynamics in tropical areas, and for the estimation of surface soil moisture in areas with sparse to no vegetation cover.

\section{RADAR TECHNOLOGY DEVELOPMENT}

We expect that the future radar missions will be noticeably different from the previous missions. Future science radar missions must cost substantially less than the current missions in order to be competitive with other remote sensing instruments. To reduce the cost of a radar mission, it is necessary to utilize advanced radar technologies for constructing the lighter weight and more power efficient radar hardware. In the next two sections, we describe advanced radar technologies that may be used for future radar missions.

\subsection{Radar antenna technology}

Since the antenna weight is usually more than half of the entire space-borne radar mass especially with the complicated deployment mechanism, the radar antenna is the prime candidate for implementing radically different technology. Phased array technology was used for the SIR$\mathrm{C}$ antennas at both $\mathrm{L}$ - and $\mathrm{C}$-band; however, the antenna was very heavy and the weight needs to be reduced significantly. The QuikScat antenna is a rotating reflector antenna with 1 meter diameter. Mechanical rotation of the antenna is required to obtain a wide swath to be useful for ocean scatterometery. Since QuikScat is a real aperture radar, the antenna size determines the achievable ground resolution. In order to improve the performance, it will be necessary to develop a large light-weight antenna.

The antenna physical size must be large enough to obtain the necessary SNR (Signal to Noise Ratio) because the transmit power is limited in the space environment. For a typical L-band SAR, the physical antenna area is approximately $30 \mathrm{~m}^{2}$. The most promising approach for significantly reducing the antenna mass is the use of space inflatable structures to deploy the antenna and to provide the antenna structure [5]. Microstrip radiation elements are etched on the conductor-dielectric layer thin-film membrane surface in order to fold it within a small volume. The expected mass density is less than $3 \mathrm{~kg} / \mathrm{m}^{2}$ which includes the aperture, structure, inflation system and $T / R$ modules. This is a significant improvement when it is compared with the SIR-C antenna density of $23 \mathrm{~kg} / \mathrm{m}^{2}$ for L-band antenna panel alone without the structure. It is also advantageous to implement a membrane $T / R$ module that can be integrated with a membrane layer. This membrane $T / R$ module must be flexible enough to be folded in a small volume.

A similar membrane radiator approach can be used for a ReflectArray antenna. Using this concept, an electrically equivalent parabolic reflector can be 
constructed by using flat membrane layers. Patch radiation elements with appropriate phase correction feeds are etched on the front membrane layer. The flatness of each membrane layer is maintained by an inflatable structure that holds all membrane layers. A $3 \mathrm{~m}$ diameter Ka-band membrane ReflectArray has been constructed and tested successfully at JPL using an inflatable structure.

Deployable light weight antennas are also promising candidates for planetary radar missions. An array of three Yagi antennas is proposed for the Europa radar sounder. The array has a maximum gain of about 12 $\mathrm{dB}$ in the nadir direction at $50 \mathrm{MHz}$. However, the total mass of the antenna is estimated to be less than $5 \mathrm{~kg}$. This antenna design was proposed by Los Alamos National Laboratory. A similar structure is also under consideration for the MARSIS antenna. Deployable mesh reflectors are also useful for various radar missions that do not require fast antenna beam steering. These mesh reflectors are attractive due to their availability at lower cost and the broadband characteristic for a multiple frequency radar mission.

For an interferometric radar mission, two antennas must be separated in space to form an interferometric baseline. Since the baseline must be known accurately, it is advantageous to use a large light-weight structure to connect two antennas rigidly. As an example, the SRTM uses a 60 meter mast extending from the shuttle payload bay. That is, one antenna is at the shuttle's payload bay and the other is at the end of the mast. The 60 meter mast forms a rigid interferometric baseline. A similar technology is also suggested for the Wide Swath Ocean Altimeter (WSOA) mission to measure the ocean topography by using radar interferometry. As radar interferometry becomes more popular, a light-weight large structure such as an inflatable structure and deployable mast will be frequently used to support two or more radar antennas.

For an airborne radar, a flat microstrip antenna is useful when it is integrated with an aircraft. One of major advantages of an airborne radar is the high resolution capability. In order to produce a high resolution radar image, it is necessary to transmit a broad band signal. This broadband capability requires an antenna with large fractional bandwidth especially at low frequencies. As an example, the GeoSAR antenna achieved a fractional bandwidth close to $50 \%$. Various broadband antennas are under development for high resolution imaging radars.

\subsection{Radar electronics technology}

The radar electronics and T/R modules have to be efficient in power consumption to reduce the weight and complexity associated with the solar panel and other power related subsystems. As an example, the future single channel L-band radar electronics consumes less than $50 \mathrm{~W}$. The radar electronic will be miniaturized by using advanced technologies such as Monolithic Microwave Integrated Circuit (MMIC), MicroElectroMechanical System (MEMS), Surface Acoustic Wave (SAW) device, Application Specific Integrated Circuit (ASIC), and Field Programmable Gate Array (FPGA) technology. The expected weight of a single channel L-band radar electronics will be less than $15 \mathrm{~kg}$ and the entire volume will be less than 10 " 12 " 19 ". The radar electronics will be designed to be easily integrated with the spacecraft hardware.

For a deep space mission such as the Europa Orbiter mission, the available power is extremely limited. Therefore, an efficient transmitter is required to operate a radar sounder. A $50 \mathrm{MHz}$, Class E amplifier is proposed for the Europa Orbiter sounder. Currently, a $100 \mathrm{~W}$ amplifier with over $80 \%$ efficiency is being studied at Caltech and JPL. This amplifier may be implemented using a combination of a Class-A preamplifier and a Class-E power amplifier. For the Europa sounder, the receiver is designed to provide extremely large dynamic range. The most difficult task in developing the Europa sounder is to build a digital subsystem that must operate in the $1 \mathrm{Mrad}$ radiation environment.

In order to reduce the complexity of a radar transmitter system, a chirp signal may be generated at the carrier frequency using an optical device. For a large phased array, an optical fiber network can be used to deliver a radar signal to antenna $T / R$ modules. When a large area is to be imaged, a digital beam forming technique can be implemented. A high frequency digital beam forming altimeter has been proposed at JPL as a Mars landing radar. Since the data volume associated with the radar instrument is extremely high, the requirement for the data downlink system is very demanding. Unlike optical images, radar raw data cannot be compressed since it is a Gaussian noise. Therefore, to reduce the downlink data rate significantly, we need to develop on-board processing technology, especially for planetary missions.

\section{SUMMARY}

To enable a low cost radar mission, various radar techniques and technologies are being developed at many institutions in the world. We summarized several research activities at JPL in this paper.

\section{REFERENCES}

[1] C. Elachi, Introduction to the Physics and Techniques 
of Remote Sensing, Wiley \& Sons, New York, 1987.

[2] J. J. van Zyl, H. A. Zebker, and C. Elachi, “ Imaging Radar Polarization Signatures: Theory and

Observation," Radio Science, Vol. 22, 529-543, 1987.

[3] H. Zebker and R. Goldstein, "Topographic Mapping from Interferometric SAR Observations," J. Geophys. Res., 91, 4993-4999, 1986.

[4] J. E. Hilland, et al., "Future NASA Spaceborne SAR Missions," IEEE-AES Systems Magazine, Vol. 13, No. 11, November 1998.

[5] J. Huang, et al., "An Inflatable L-band Microstrip SAR Array," IEEE APS/URSI Symposium, Atlanta, Georgia, June 1998.

\section{ACKNOWLEDGMENT}

The research described in this paper was carried out by the Jet Propulsion Laboratory, California Institute of Technology, under a contract with the National Aeronautics and Space Administration. 